\title{
Stable breathing through deeper sleeping
}

\author{
Julian Saboisky, Danny Eckert, Atul Malhotra
}

\begin{abstract}
Although there is evidence that patients with obstructive sleep apnoea (OSA) have anatomical susceptibility coupled with abnormalities in upper airway motor control and/or instability in ventilatory control, the data now suggest that the mechanisms underlying OSA vary considerably between patients. ${ }^{1}$ In some patients OSA is primarily the result of anatomical problems, while in others it may be due to dysfunctional motor control with only minimal anatomical abnormality. Similarly, abnormal ventilatory control (loop gain) may be important in only a subgroup of patients with OSA. ${ }^{2}$ As a result, the concept of individualised therapy has now emerged, such that treatment of the major underlying abnormality is likely to be beneficial in the appropriately targeted patient subgroups. ${ }^{3}$ In theory, patients with OSA who respond well to palatal surgery probably primarily have an abnormality in the velopharyngeal anatomy, ${ }^{4}$ whereas those who respond well to oxygen may have mainly an abnormality in ventilatory control instability. 56
\end{abstract}

The arousal threshold is one concept that has received some-but not sufficient-attention in the OSA arena. ${ }^{7}$ The trigger for arousal from sleep during respiratory events is believed to be increasing negative intrathoracic pressure ${ }^{9}$ (see figure 1). The realisation of the importance of the arousal threshold in the pathogenesis of OSA has recently been brought to light by the work of Younes, particularly the emphasis that most patients with OSA spontaneously have some periods of stable breathing. ${ }^{10} 11$ Jordan et al recently observed that these stable breathing periods were associated with markedly increased activity in the genioglossus, suggesting that upper airway dilator muscles are necessary and sufficient to stabilise the upper airway with endogenous stimuli. ${ }^{12}$ Upper airway muscles are known to have robust responsiveness to

Brigham and Women's Hospital and Harvard Medical School, Boston, Massachusetts, USA

Correspondence to Atul Malhotra, Sleep Disorders Research Program @ BIDMC, Brigham and Women's Hospital and Harvard Medical School, 75 Francis Street, Boston, MA 02115, USA; amalhotra1@partners.org respiratory stimuli including $\mathrm{CO}_{2}$ (through chemoreceptors) and through intrapharyngeal negative pressure (via mechanoreceptors) during wakefulness. ${ }^{13} 14$ Recent single motor unit studies of the genioglossus suggest that recruitment is likely to be mediated through activity in additional motor units rather than significant increases in motor unit firing frequency, although further work is required in this area. ${ }^{15}$ However, during sleep the responsiveness of these muscles is considerably attenuated compared with wakefulness, such that more marked stimuli for greater duration are required to achieve upper airway muscle recruitment. ${ }^{16}$ Because accumulation of respiratory stimuli takes time to occur during sleep, the arousal threshold becomes a critical variable-that is, premature arousal from sleep may lead to repetitive apnoea due to inadequate accumulation of respiratory stimuli to activate upper airway muscles. On the other hand, a very high arousal threshold (hard to wake up) may be deleterious if profound hypoxaemia and hypercapnia occur before arousal. Thus, manipulation of the arousal threshold may be beneficial for some patients (those with a low arousal threshold but recruitable upper airway muscles) (figure 1) but deleterious for others (in whom deterioration in blood gases leads to end organ damage).

Several points are worthy of emphasis from the standpoint of arousal. First, recent studies have shown that the increase in arousal threshold seen in patients with OSA is probably acquired (at least in part) rather than intrinsic to the disease. ${ }^{17}$ Thus, with continuous positive airway pressure (CPAP) the arousal threshold in OSA is lowered, suggesting that the increase in arousal threshold is probably an adaptive response which occurs over time in response to sleep fragmentation and/or hypoxaemia. Second, certain hypnotic agents can raise the arousal threshold and are likely to be beneficial for carefully selected patients with $\mathrm{OSA}^{18}$ (figure 1). Third, when arousal occurs, there is a robust ventilatory response which itself can be destabilising. ${ }^{19}$ If the ventilatory response to arousal is sufficiently marked, it can drive the arterial carbon dioxide tension below the chemical apnoea threshold, leading to either central or obstructive apnoea depending on the prevailing upper airway mechanics. Of note, many techniques that assess ventilatory instability rely on stable state (no arousals), such that the impact of the ventilatory response to arousal is frequently overlooked. ${ }^{20}$

In this issue of Thorax, Ratnavadival et $a l^{21}$ provide an important insight into several critical questions (see page 107). The authors have observed an increase in arousal threshold to respiratory stimuli in slow wave sleep (SWS) compared with stage 2 non-REM sleep. This concept has been minimally studied but is consistent with changes observed between stage 2 and SWS in healthy men in response to occlusion stimuli. ${ }^{22}$ A separate study reported data for minimal oesophageal pressure before arousal during naturally occurring events in light sleep (stages 1 and 2) and SWS in patients with severe OSA. ${ }^{23}$ However, no direct comparisons were made and subject numbers differed, precluding retrospective comparison of these data between light sleep and SWS. Thus, the observations by Ratnavadival et al help answer an age-old question as to why SWS is stabilising from the standpoint of breathing. While some have argued that SWS only occurs when breathing is stable, others have suggested that SWS per se stabilises breathing. The observation that SWS is associated with an increase in arousal threshold might suggest that patients with OSA experience stable breathing due to an accumulation of respiratory stimuli rather than SWS being simply a marker of stable breathing. Some but not all studies have shown increased phasic upper airway muscle activity in SWS compared with other non-REM stages. ${ }^{24}$ The new findings have important clinical implications and might suggest that, if a high arousal threshold could be achieved pharmacologically, a stable breathing pattern may be predicted. Such an approach would have to be undertaken cautiously since the selection of patients and the appropriate agent for this approach would be critical. In theory, a non-myorelaxant hypnotic would be predicted to stabilise breathing if given to patients with a low arousal threshold but with recruitable upper airway muscles (figure 1). Alternatively, certain agents have been reported to induce SWS (eg, sodium oxybate, ${ }^{25}$ thalidomide, high-dose trazodone ${ }^{26}$ ), and they may also theoretically provide benefits from the standpoint 


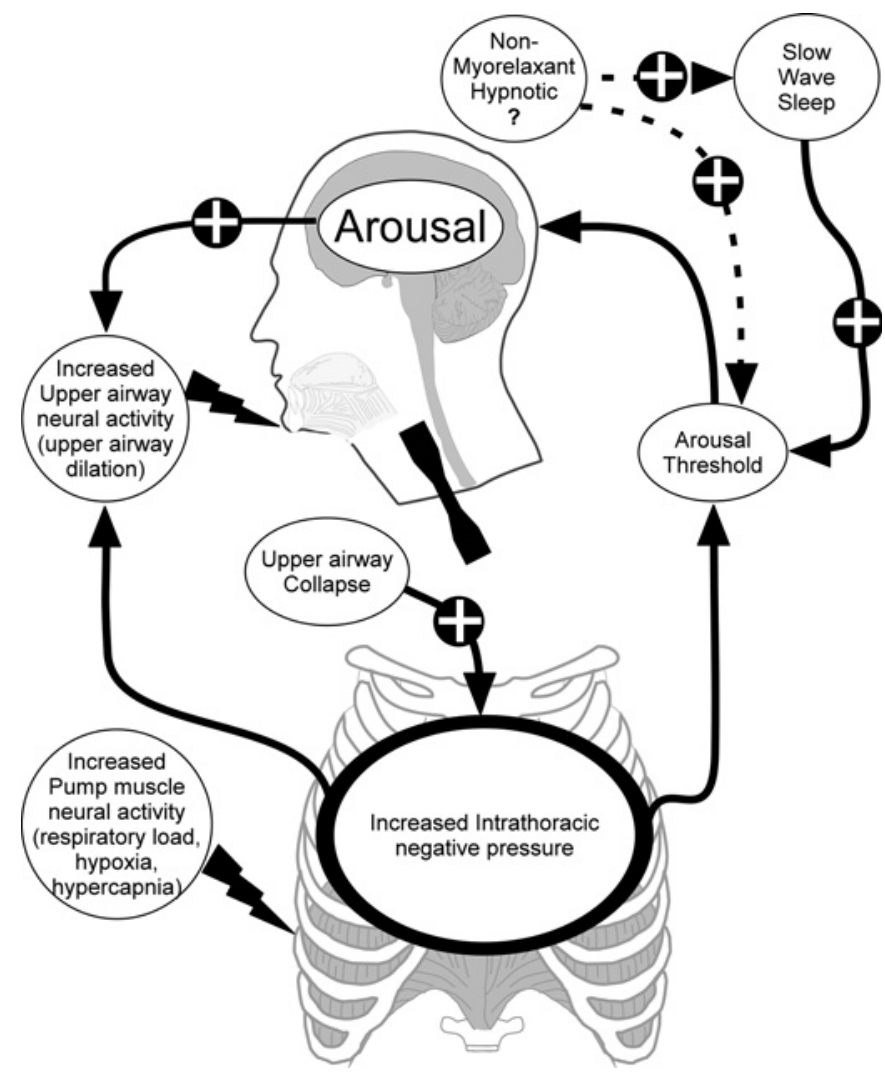

Figure 1 Schematic representation of the chain of events that leads to arousal in obstructive sleep apnoea and possible modulating factors. The stimulus to respiratory load-induced arousal is believed to be increased respiratory effort (increased intrathoracic negative pressure). In the case of obstructive sleep apnoea, this is typically associated with hypoxia or hypercapnia. These stimuli provide additional neural output to the respiratory pump and upper airway dilator muscles. The termination of an apnoea or hypopnoea generally requires arousal from sleep. However, if upper airway dilator muscle activity increases sufficiently to re-establish pharyngeal patency, the event may be terminated without an arousal and thus less sleep fragmentation. Slow wave sleep increases the arousal threshold, thus potentially providing additional time for the upper airway muscles to respond sufficiently to overcome the collapsed pharynx. Non-myorelaxant hypnotics may directly increase the arousal threshold or slow wave sleep and promote stable breathing (proposed pathways indicated by dotted lines). In selected patients (with a low arousal threshold), this pharmacological approach may be beneficial. The plus sign indicates increased input to the selected pathway. Some arrows have been deleted for clarity.

of breathing. However, blinded randomised trials will clearly be required to determine whether these strategies actually improve clinical outcomes. In theory, the markedly negative intrathoracic pressure seen during stable flow limited breathing may represent a substantial afterload on the left ventricle. ${ }^{27} 28$ Such an afterload effect could potentially outweigh the benefits of reducing hypoxaemia and arousals in certain patients. Ultimately, genetic and other biomarkers may be required to determine to which physiological stress a given individual may be most susceptible and from what pharmacological strategy an individual may benefit.

\section{Competing interests None.}

Provenance and peer review Commissioned; not externally peer reviewed.
Thorax 2010;65:95-96.

doi:10.1136/thx.2009.127860

\section{REFERENCES}

1. Eckert DJ, Malhotra A. Pathophysiology of adult obstructive sleep apnea. Proc Am Thorac Soc 2008:5:144-53.

2. Wellman A, Jordan AS, Malhotra A, et al. Ventilatory control and airway anatomy in obstructive sleep apnea. Am J Respir Crit Care Med 2004;170:1225-32.

3. Saboisky JP, Chamberlin NL, Malhotra A. Potential therapeutic targets in obstructive sleep apnoea. Expert Opin Ther Targets 2009;13:795-809.

4. Isono S, Shimada A, Tanaka A, et al. Effects of uvulopalatopharyngoplasty on collapsibility of the retropalatal airway in patients with obstructive sleep apnea. Laryngoscope 2003:113:362-7.

5. Wellman A, Malhotra A, Jordan AS, et al. Effect of oxygen in obstructive sleep apnea: role of loop gain. Respir Physiol Neurobiol 2008:162:144-51.

6. Smith PL, Haponik EF, Bleecker ER. The effects of oxygen in patients with sleep apnea. Am Rev Respir Dis 1984;130:958-63.
7. Berry RB, Kouchi KG, Der DE, et al. Sleep apnea impairs the arousal response to airway occlusion. Chest 1996;109:1490-6.

8. Younes M. Role of arousals in the pathogenesis of obstructive sleep apnea. Am J Respir Crit Care Med 2004; 169:623-33.

9. Gleeson K, Zwillich CW, White DP. The influence of increasing ventilatory effort on arousal from sleep. $A m$ Rev Respir Dis 1990;142:295-300.

10. Younes M. Role of respiratory control mechanisms in the pathogenesis of obstructive sleep disorders. J Appl Physiol 2008:105:1389-405.

11. Younes M, Ostrowski M, Atkar R, et al. Mechanisms of breathing instability in patients with obstructive sleep apnea. J Appl Physiol 2007;103:1929-41.

12. Jordan AS, White DP, Lo YL, et al. Airway dilator muscle activity and lung volume during stable breathing in obstructive sleep apnea. Sleep 2009;32:361-8.

13. Lo YL, Jordan AS, Malhotra A, et al. Genioglossal muscle response to $\mathrm{CO}_{2}$ stimulation during NREM sleep. Sleep 2006;29:470-7.

14. Horner RL, Innes JA, Murphy K, et al. Evidence for reflex upper airway dilator muscle activation by sudden negative airway pressure in man. $J$ Physiol (Lond) 1991;436:15-29.

15. Wilkinson V, Malhotra A, Nicholas C, et al. Discharge patterns of human genioglossus motor units during arousal from sleep. Sleep 2008;31:525-33.

16. Stanchina M, Malhotra A, Fogel RB, et al. Genioglossus muscle responsiveness to chemical and mechanical loading during NREM sleep. Am J Respir Crit Care Med 2002;165:945-9

17. Loewen A, Ostrowski M, Laprairie J, et al. Determinants of ventilatory instability in obstructive sleep apnea: inherent or acquired? Sleep 2009; 32:1355-65

18. Heinzer RC, White DP, Jordan AS, et al. Trazodone increases arousal threshold in obstructive sleep apnoea. Eur Respir J 2008;31:1308-12.

19. Jordan A, McEvoy R, Edwards J, et al. The influence of gender and upper airway resistance on the ventilatory response to arousal in obstructive sleep apnea. J Physiol 2004;558:993-1004.

20. Meza S, Giannouli E, Younes M. Control of breathing during sleep assessed by proportional assist ventilation. J Appl Physiol 1998;84:3-12.

21. Ratnavadivel R, Stadler D, Windler S, et al. Upper airway function and arousability to ventilatory challenge in slow wave versus stage 2 sleep in obstructive sleep apnoea. Thorax 2010;65:107-12.

22. Berry RB, Bonnet MH, Light RW. Effect of ethanol on the arousal response to airway occlusion during sleep in normal subjects. Am Rev Respir Dis 1992;145/(2 Pt 1):445-52.

23. Pelin Z, Karadeniz D, Ozturk L, et al. The role of mean inspiratory effort on daytime sleepiness. Eur Respir $J$ 2003;21:688-94.

24. Basner RC, Ringler J, Schwartzstein RM, et al. Phasic electromyographic activity of the genioglossus increases in normals during slow-wave sleep. Respir Physiol 1991;83:189-200.

25. Series F, Series I, Cormier Y. Effects of enhancing slow-wave sleep by gamma-hydroxybutyrate on obstructive sleep apnea. Am Rev Respir Dis 1992:145:1378-83.

26. Veasey SC, Fenik P, Panckeri K, et al. The effects of trazodone with L-tryptophan on sleep-disordered breathing in the English bulldog. Am J Respir Crit Care Med 1999:160((5 Pt 1):1659-67.

27. Fessler $\mathbf{H}$, Brower $\mathrm{R}$, Wise $\mathrm{R}$, et al. Mechanism of reduced LV afterload by systolic and diastolic positive pleural pressure. J Appl Physiol 1988;65:1244-50.

28. Malhotra A, Muse VV, Mark EJ. Case records of the Massachusetts General Hospital. Weekly clinicopathological exercises. Case 12-2003. An 82-year-old man with dyspnea and pulmonary abnormalities. N Engl J Med 2003;348:1574-85. 